\title{
A Protective Role of FAM13A in Human Airway Epithelial Cells Upon Exposure to Cigarette Smoke Extract
}

\author{
Qing Chen 1,2*, Maaike de Vries' ${ }^{2,3}$, Kingsley Okechukwu Nwozor ${ }^{1,2}$, \\ Jacobien A. Noordhoek ${ }^{1,2,4}$, Corry-Anke Brandsma ${ }^{1,2}$, H. Marike Boezen ${ }^{2,3}$ and \\ Irene $H$. Heijink $k^{1,2,4}$
}

\begin{abstract}
${ }^{1}$ Department of Pathology and Medical Biology, University Medical Center Groningen, University of Groningen, Groningen, Netherlands, ${ }^{2}$ Groningen Research Institute for Asthma and COPD (GRIAC), University Medical Center Groningen, University of Groningen, Groningen, Netherlands, ${ }^{3}$ Department of Epidemiology, University Medical Center Groningen, University of Groningen, Groningen, Netherlands, ${ }^{4}$ Department of Pulmonology, University Medical Center Groningen, University of Groningen, Groningen, Netherlands
\end{abstract}

\section{OPEN ACCESS}

Edited by:

Rosalinda Sorrentino,

University of Salerno, Italy

Reviewed by:

Gert Folkerts,

Utrecht University, Netherlands

Massimo Caruso,

University of Catania, Italy

*Correspondence:

Qing Chen

q.chen@umcg.nl

Specialty section: This article was submitted to

Respiratory Physiology, a section of the journal

Frontiers in Physiology

Received: 04 April 2021 Accepted: 11 May 2021

Published: 07 June 2021

Citation:

Chen $Q$, de Vries M, Nwozor KO,

Noordhoek JA, Brandsma CA,

Boezen HM and Heijink IH (2021) A Protective Role of FAM13A in Human Airway Epithelial Cells Upon Exposure

to Cigarette Smoke Extract.

Front. Physiol. 12:690936.

doi: 10.3389/fphys.2021.690936
Background: Chronic Obstructive Pulmonary Disease (COPD) is a progressive lung disease characterized by chronic inflammation upon inhalation of noxious particles, e.g., cigarette smoke. FAM13A is one of the genes often found to be associated with COPD, however its function in the pathophysiology of COPD is incompletely understood. We studied its role in airway epithelial barrier integrity and cigarette smoke-induced epithelial responses.

Materials and Methods: Protein level and localization of FAM13A was assessed with immunohistochemistry in lung tissue from COPD patients and non-COPD controls. In vitro, FAM13A expression was determined in the absence or presence of cigarette smoke extract (CSE) in primary airway epithelial cells (AECs) from COPD patients and controls by western blotting. FAM13A was overexpressed in cell line 16HBE140- and its effect on barrier function was monitored real-time by electrical resistance. Expression of junctional protein E-cadherin and $\beta$-catenin was assessed by western blotting. The secretion of neutrophil attractant CXCL8 upon CSE exposure was measured by ELISA.

Results: FAM13A was strongly expressed in airway epithelium, but significantly weaker in airways of COPD patients compared to non-COPD controls. In COPD-derived AECs, but not those of controls, FAM13A was significantly downregulated by CSE. 16HBE140- cells overexpressing FAM13A built up epithelial resistance significantly more rapidly, which was accompanied by higher E-cadherin expression and reduced CSE-induced CXCL8 levels.

Conclusion: Our data indicate that the expression of FAM13A is lower in airway epithelium of COPD patients compared to non-COPD controls. In addition, cigarette smoking selectively downregulates airway epithelial expression of FAM13A in COPD patients. This may have important consequences for the pathophysiology of COPD, as the more rapid build-up of epithelial resistance upon FAM13A overexpression suggests improved (re)constitution of barrier function. The reduced epithelial secretion of CXCL8 upon CSE-induced damage suggests that lower FAM13A expression upon cigarette smoking may facilitate epithelial-driven neutrophilia.

Keywords: COPD-Chronic obstructive pulmonary disease, FAM13A, airway epithelium, barrier function, CXCL8 


\section{INTRODUCTION}

Chronic Obstructive Pulmonary Disease (COPD) is a severe and debilitating inflammatory lung disease characterized by persistent airflow limitation and reduced gas exchange. Patients with COPD suffer from severe respiratory symptoms such as chronic cough and shortness of breath, which negatively influence their quality of life (Barnes et al., 2003). It is estimated that 384 million people suffered from COPD in 2010 and COPD is expected to become the third leading cause of death (Adeloye et al., 2015). Important features of COPD are neutrophilic inflammation in the lungs, mucus hypersecretion in the large airways, and abnormal tissue damage and repair responses resulting in (small) airway remodeling and destruction of alveolar tissue.

One of the main risk factors for the development of COPD is exposure to cigarette smoke, which first encounters the airway epithelial cells (AECs) (van der Vaart et al., 2005; Abrams et al., 2014). AECs maintain barrier integrity by forming intercellular contacts, including adherens junctions and tight junctions, to defend the underlying cells from inhaled insults (Hellings and Steelant, 2020). Cigarette smoke causes mitochondrial damage and oxidative stress (Aghapour et al., 2020), leading to epithelial damage, affecting epithelial intercellular contacts (Aghapour et al., 2018). In vitro, cigarette smoke extract (CSE) disrupts epithelial junctions, of which E-cadherin is a key constituent (Heijink et al., 2012), being linked to the cytoskeleton by $\beta$-catenin. In COPD, the airway epithelium may be less able to recover from this type of damage, as persistent disruption of airway epithelial cell-cell contacts has been observed in COPD (Oldenburger et al., 2014) together with the inability to form cell-cell contacts, polarize and (re)differentiate in vitro (Heijink et al., 2014). Defective epithelial barrier function not only leads to increased permeability to inhaled particles (Gangl et al., 2009; Heijink et al., 2012) it also promotes pro-inflammatory activity of the epithelium (Aghapour et al., 2018). AECs, especially when damaged, secrete pro-inflammatory mediators such as CXCL8, which recruits neutrophils (Higham et al., 2018). Repeated injury of the airway epithelium by inhaled particles, such as cigarette smoke, may result in chronic inflammation in susceptible individuals and ultimately lead to chronic bronchitis and/or emphysema in COPD (Barnes et al., 2003; Barnes, 2008; Brusselle et al., 2011).

Although the pathogenesis of COPD is still not fully understood, it is known that its development is influenced by both genetic and environmental factors. Why the airway epithelium of certain individuals is more susceptible to abnormal pro-inflammatory, damage, and repair responses to cigarette smoke is currently unknown. To unravel this individual susceptibility, large scale genome-wide association studies (GWAS) on COPD have been performed, resulting in the identification of multiple susceptibility genes for COPD (Hobbs et al., 2017; van der Plaat et al., 2017). Of interest, these susceptibility genes are often expressed in the airway epithelium. One of such a susceptibility gene is Family with Sequence Similarity 13 A (FAM13A) (Jiang et al., 2016), which was shown to be involved in emphysema susceptibility, and to regulate mitochondrial function (Jiang et al., 2016, 2017; Hawkins and Mora, 2017), and $\beta$-catenin stability (Meng and Takeichi, 2009; Jiang et al., 2016). Jiang and co-workers also showed that FAM13A is expressed in lung tissue (Jiang et al., 2016), however they did not study its localization within the tissue. Moreover, it is not understood how altered expression or function contributes to the development of COPD. We hypothesized that FAM13A affects the susceptibility to cigarette smoke in airway epithelial cells. Therefore, in this study we investigated the expression of FAM13A in lung tissue, the effect of cigarette smoke exposure on airway epithelial FAM13A expression and its functional role in airway epithelial barrier function and pro-inflammatory responses in human AECs.

\section{MATERIALS AND METHODS}

\section{Subjects and Design of the Study}

Human lung tissue for immunohistochemical staining was derived from 11 GOLD stage IV COPD patients, and 9 nonCOPD controls (see Table 1 for subject characteristics). Primary AECs were isolated and cultured from tracheobronchial tissue of 9 GOLD stage III and IV COPD patients undergoing lung transplantation (see Table 2 for subject characteristics). The study protocol was consistent with the Research Code of the University Medical Center Groningen ${ }^{1}$ and national ethical and professional

\footnotetext{
${ }^{1}$ https://www.umcg.nl/SiteCollectionDocuments/English/Researchcode/umcgresearch-code-2018-en.pdf
}

TABLE 1 | Characteristics of the subjects from whom lung tissue was obtained

\begin{tabular}{lcc}
\hline & Control & COPD IV \\
\hline Subjects, N & 9 & 12 \\
Female, N & 6 & 8 \\
Age, years (range) & $55(43-62)$ & $52(47-55)$ \\
Smoking status & Ex-smoker & Ex-smoker \\
FEV1 \% predicted & $104(85-127)$ & $17.4(12-29.9)$
\end{tabular}

Data are presented as median (range) unless otherwise stated. FEV1: forced expiratory volume in $1 \mathrm{~s}$, the best one among multiple measurements is shown. COPD patients were included based on the Global Initiative for Chronic Obstructive Lung Disease standard.

TABLE 2 | Characteristics of COPD patients from whom airway epithelial cells (AECs) were obtained.

\begin{tabular}{lcccccc}
\hline Age Gender & $\begin{array}{c}\text { Smoking } \\
\text { status }\end{array}$ & Packs/year & $\begin{array}{c}\text { FEV1 } \\
\text { (\%predicted) }\end{array}$ & FEV1/FVC & $\begin{array}{c}\text { COPD } \\
\text { Stage }\end{array}$ \\
\hline 60 & F & EX & 38 & 18 & 18 & IV \\
54 & F & EX & 35 & 25 & 28 & IV \\
56 & M & EX & 30 & 31 & 29 & III \\
63 & F & EX & 40 & 22 & 32 & IV \\
63 & M & NS & 0 & 41 & 45 & III \\
63 & M & EX & 45 & 6 & 40 & IV \\
51 & M & EX & 30 & 16 & 32 & IV
\end{tabular}

M, male; F, female; EX, ex-smoker; NS, never smoker; FEV1, forced expiratory volume in $1 \mathrm{~s}$; FVC, forced vital capacity. 
guidelines ("Code of conduct; Dutch federation of biomedical scientific societies," ${ }^{2}$ ). Control-derived AECs were obtained from the leftover tracheobronchial tissue of 10 transplantation donor lungs without COPD from whom no information was available. Airway epithelial cultures were obtained by enzymatic treatment and stored in liquid nitrogen before experimentation as described previously (Heijink et al., 2016).

\section{Immunohistochemistry Staining}

Localization and expression of FAM13A protein was determined using immunohistochemical staining in lung tissue. Lung tissue was embedded in paraffin and cut into three- $\mu \mathrm{m}$ thick lung sections. Antigen retrieval was done with $10 \mathrm{mM}$ citrate buffer at $\mathrm{pH} 6.0$ followed by avidin/biotin blocking using the Avidin/Biotin blocking kit (Vector Laboratories, Inc., Burlingame, CA 94010). Specific primary antibody against FAM13A (55401-1-AP, Proteintech, Manchester, United Kingdom), E-cadherin (610182, BD Bioscience, Breda, NL), secondary antibody Goat AntiRabbit Immunoglobulins/HRP (Dako P0488, Amsterdam, NL), Rabbit Anti-Mouse Immunoglobulins/HRP (Dako P0260, Amsterdam, NL) and tertiary antibody Streptavidin/HRP (Dako P0397, Canada) were used. Positive staining was visualized using VECTOR ${ }^{\circledR}$ NovaRED $^{\mathrm{TM}}$ (SK-4800, Vector Laboratories, Canada). The slides were scanned with the Hamamatsu NanoZoomer 2.0HT digital slide scanner, and representative pictures were taken using the Hamamatsu NDP view 2 software.

\section{Analysis of Immunohistochemistry Staining}

The epithelial layer was extracted from airways using Adobe Photoshop (Adobe Inc. California, United States) and ImageJ (Bethesda, Maryland, United States ${ }^{3}$ ) was used to quantify the intensity and distribution of the staining (Schindelin et al., 2012). NovaRed staining in the images was measured by isolating the signal with color deconvolution plugin by Gabriel Landini (Ruifrok and Johnston, 2001). Thresholding was then used to select the positive pixels, presenting the data as the average intensity of the NovaRed stain of all airways per donor.

\section{Epithelial Cell Culture}

COPD and control-derived primary AECs were derived from fresh bronchial tissue using enzymatic treatment, stored in liquid nitrogen and cultured as described previously (Heijink et al., 2016). In short, cells were seeded into T25 culture flasks coated with $30 \mu \mathrm{g} / \mathrm{ml}$ collagen, $30 \mu \mathrm{g} / \mathrm{ml}$ fibronectin and $10 \mu \mathrm{g} / \mathrm{ml}$ BSA in hormonally supplemented bronchial epithelium growth medium (BEGM; Lonza) with $100 \mathrm{U} / \mathrm{ml}$ Penicillin and $100 \mathrm{mg} / \mathrm{ml}$ streptomycin. When $90 \%$ confluent, the cells were expanded and seeded in duplicates for experiments at passage 3 .

The human bronchial epithelial cell line 16HBE140- was generously offered by D.C. Gruenert (University of California, San Francisco, CA, United States) and the cells were cultured

${ }^{2}$ www.federa.org

${ }^{3}$ http://rsb.info.nih.gov/ij/download.html as described previously (Heijink et al., 2007). Briefly, cells were cultured in T25 flasks (Corning, NY, United States) or 24-wells plates coated with $30 \mu \mathrm{g} / \mathrm{ml}$ collagen and 10 $\mu \mathrm{g} / \mathrm{ml}$ bovine serum albumin (BSA) in EMEM (Biowhittaker, Verviers, Belgium) with $2 \mathrm{mM}$ GlutaMax (Life Technologies, Paisley, United Kingdom), $100 \mathrm{U} / \mathrm{ml}$ Penicillin and $100 \mathrm{mg} / \mathrm{ml}$ streptomycin, and $10 \%$ fetal calf serum (FCS; Biowhittaker, Verviers, Belgium). When $90 \%$ confluent, the cells were passaged or seeded in duplicates into coated 24-wells or 12-wells plates for experiments.

\section{Cigarette Smoke Extract Preparation}

Kentucky 3R4F research-reference cigarettes (The Tobacco Research Institute, University of Kentucky, Lexington, KY, United States) were used to prepare CSE. Filters were removed and the cigarettes were smoked using a hose pump (Watson Marlow 603S, Cornwall, United Kingdom) at a rate of 7 $\mathrm{L} / \mathrm{h}$ (Pouwels et al., 2016). The smoke of two cigarettes was bubbled through $25 \mathrm{~mL}$ EMEM medium and defined as $100 \%$ CSE. The extract was used freshly within $15 \mathrm{~min}$ after preparation.

\section{Treatment of the Cells}

For CSE exposure, cells were seeded into 24-wells plates at a density of $5 \times 10^{4}$ cells/well, grown for 2-3 days until $90 \%$ confluence was reached, and hormonally-deprived (AECs) overnight. Next, cells were stimulated with 0,10 or $20 \%$ CSE or EMEM/BEBM alone as control, for 6 or $24 \mathrm{~h}$ before harvesting for RNA isolation, cell lysate preparation and collection of the supernatant.

For FAM13A functional studies, a FAM13A overexpression plasmid was created with pcDNA3.1 backbones and FAM13A clones (NM_001265578.1) by GenScript (Nanjing, China) and plasmid pCMV6-6XL was used as empty vector control. $16 \mathrm{HBE} 140$ - cells were seeded in $24-$ wells plates at a density of $1 \times 10^{5}$ cells/well. The second day, the cells were transfected with $250 \mathrm{ng} /$ well plasmids using $1 \mu \mathrm{l} /$ well Lipofectamine 2000 transfection reagent in $500 \mu \mathrm{l}$ OPTIMEM (Life Technologies). After $4 \mathrm{~h}$, the transfection solution was replaced by culture medium. Cells were serum deprived overnight the second day after transfection and treated with CSE as described above.

\section{Total RNA Extraction and Real-Time PCR}

Total RNA of the cells was harvested using TriZol solution (MRC, Cincinnati, United States), RNA was isolated using chloroform and isopropanol, and RNA concentration was measured using a Nanodrop-1000 (ND 2.0; NanoDrop Technologies, Wilmington, DE, United States). One microgram of total RNA from each sample was reverse-transcribed into cDNA using the iScript cDNA synthesis kit (BIO-RAD) according to the manufacturer's protocol.

qPCR was performed in duplicates with GoTaq(R) Probe qPCR Master Mix kits (Promega Benelux, Leiden) using QuantStudio (Thermo Fisher Scientific, Waltham, United States). B2M and PPIA were used to FAM13A correct for all Taqman 
probes. FAM13A (Hs00208453_m1), B2M (Hs99999907_m1), and PPIA (Hs99999904_m1) were purchased from Thermo Fisher Scientific. Data were expressed as $2^{-\Delta \mathrm{Ct}}$ relative to the appropriate control.

\section{Western Blotting}

Total cell lysates were prepared as described previously (Heijink et al., 2007). Immunodetection was performed using anti-FAM13A (1:1,000; 55401-1-AP, Proteintech, Manchester, United Kingdom), anti- $\beta$-catenin (1:1,000; \#9587, CST, Leiden, Netherlands), Purified Mouse Anti-E-Cadherin (1:1,000; 610182, BD Transduction Laboratories ${ }^{\mathrm{TM}}$, United Stated), anti- $\beta$-actin (1:1,000; Santa Cruz Biotechnology, Heidelberg, Germany), polyclonal goat anti-rabbit and rabbit anti-mouse immunoglobulins/HRP (1:2,000; Dako Denmark, Flostrup, Denmark). The blots were developed using SuperSignal ${ }^{\mathrm{TM}}$ West Pico PLUS Chemiluminescent Substrate according to the manufacturer's guidelines (Thermo Fisher Scientific). Quantification of the western blot was performed using software Image Lab (Bio-Rad Laboratories, California, United States).

\section{ELISA}

Supernatant was centrifuged at $500 \mathrm{~g}$ for $5 \mathrm{~min}$ to remove the cells debris, and aliquoted and stored at -80 degrees. CXCL8 levels were measured in the stored cell-free supernatant using a Duoset ELISA Development Kit (DY208, R and D Systems, Minneapolis, United States), according to the manufacturer's instructions.

\section{Electric Cell-Substrate Impedance Sensing (ECIS)}

ECIS $^{\circledR}$ instrumentation (Applied Biophysics, Troy, NY, United States) was used to determine the effect of FAM13A on the formation of cell-cell contacts. In brief, 16HBE14ocells were seeded in 12-wells plates at a density of $2 \times 10^{5}$ cells/well and transfected with $500 \mathrm{ng} /$ well FAM13A plasmids as described above. ECIS arrays (8W10E, Applied Biophysics) were stabilized and coated with collagen and BSA overnight at $37^{\circ} \mathrm{C}, 5 \% \mathrm{CO}_{2}$. The second day, cells were trypsinized and re-seeded into ECIS arrays at a density of 75,000 cells/array well. We measured low-frequency resistance at $400 \mathrm{~Hz}$ as the most sensitive parameter for cell-cell contacts and high-frequency capacitance at $32,000 \mathrm{~Hz}$ as the most sensitive parameter for cell-substrate contacts and monitored cells for 3 days (Wegener et al., 2000; Heijink et al., 2010a).

\section{Statistics}

To test for differences between the conditions within groups, we applied the Wilcoxon signed-rank. For differences between groups, we used the Mann-Whitney test. Given the normal distribution in immunohistochemistry data, the Unpaired $t$-test was used here. The correlation between expression of E-cadherin and FAM13A was tested using the Pearson's correlation test. A $p<0.05$ was considered significant, tested 2-sided.

\section{RESULTS}

\section{FAM13A Expression in COPD and Control Lung Tissue and Airway Epithelial Cells (AECs)}

We first assessed which lung cell types express FAM13A using an online data tool, the human lung atlas containing single cell sequencing data (Vieira Braga et al., 2019). We observed strong expression of FAM13A in the airway epithelium, mainly ciliated cells. Additionally, fibroblasts and neutrophil subsets substantially expressed FAM13A (Figure 1A). Next, we assessed protein expression of FAM13A in lung tissue of COPD patients and non-COPD control by immunohistochemistry. In line with the gene expression, FAM13A protein was strongly expressed in the airway epithelium, and also detected in infiltrated immune cells and stromal cells, including smooth muscle (Figure 1B). The intensity of FAM13A expression was not different when analyzing whole lung tissue, but importantly, it was significantly lower in airway epithelium of COPD patients compared to in controls (Figure 1C).

In cultured AECs from COPD patients and non-COPD controls, FAM13A protein levels were not significantly different between COPD patients and non-COPD controls (Figure 2A) at baseline. Next, we exposed the cells to CSE in a concentration of $20 \%$, as was previously used to induce pro-inflammatory responses without causing cell death (Di Vincenzo et al., 2018). Here, FAM13A levels were significantly decreased upon 24-h exposure to 20\% CSE in COPD-derived primary human AECs, but not in control-derived AECs (Figure 2B).

\section{Expression of FAM13A in Human Bronchial Epithelial 16HBE140-Cells}

To study the functional role of FAM13A in airway epithelial cells, we overexpressed FAM13A in 16HBE14o- cells. We have previously validated the use of this cell line as model for primary AECs (Heijink et al., 2010a, 2013). FAM13A was overexpressed upon transfection, as confirmed by the increased FAM13A expression at mRNA and protein level already after 24 h (Figure 3). Overexpression of FAM13A was not affected by exposure to 10\% CSE (Supplementary Figure 1).

\section{Increased Electrical Resistance upon FAM13A Overexpression in 16HBE140- Cells}

To investigate if altered FAM13A expression may contribute to abnormalities observed in airway epithelial integrity in COPD, we studied whether FAM13A overexpression alters epithelial barrier function by monitoring the formation of cell-cell contacts in 16HBE14o- cells using ECIS. As observed by the stabilization of capacitance, cells reached confluence at $\sim 24 \mathrm{~h}$ after seeding (Figure 4A). After $24 \mathrm{~h}$, we observed a strong increase in resistance, which reached a plateau at $\sim 72 \mathrm{~h}$ (Figure $4 \mathrm{~B}$ ). In cells overexpressing FAM13A, resistance increased more rapidly compared to empty vector-transfected cells as reflected by the significant increase in area under the curve (Figure 4C). 

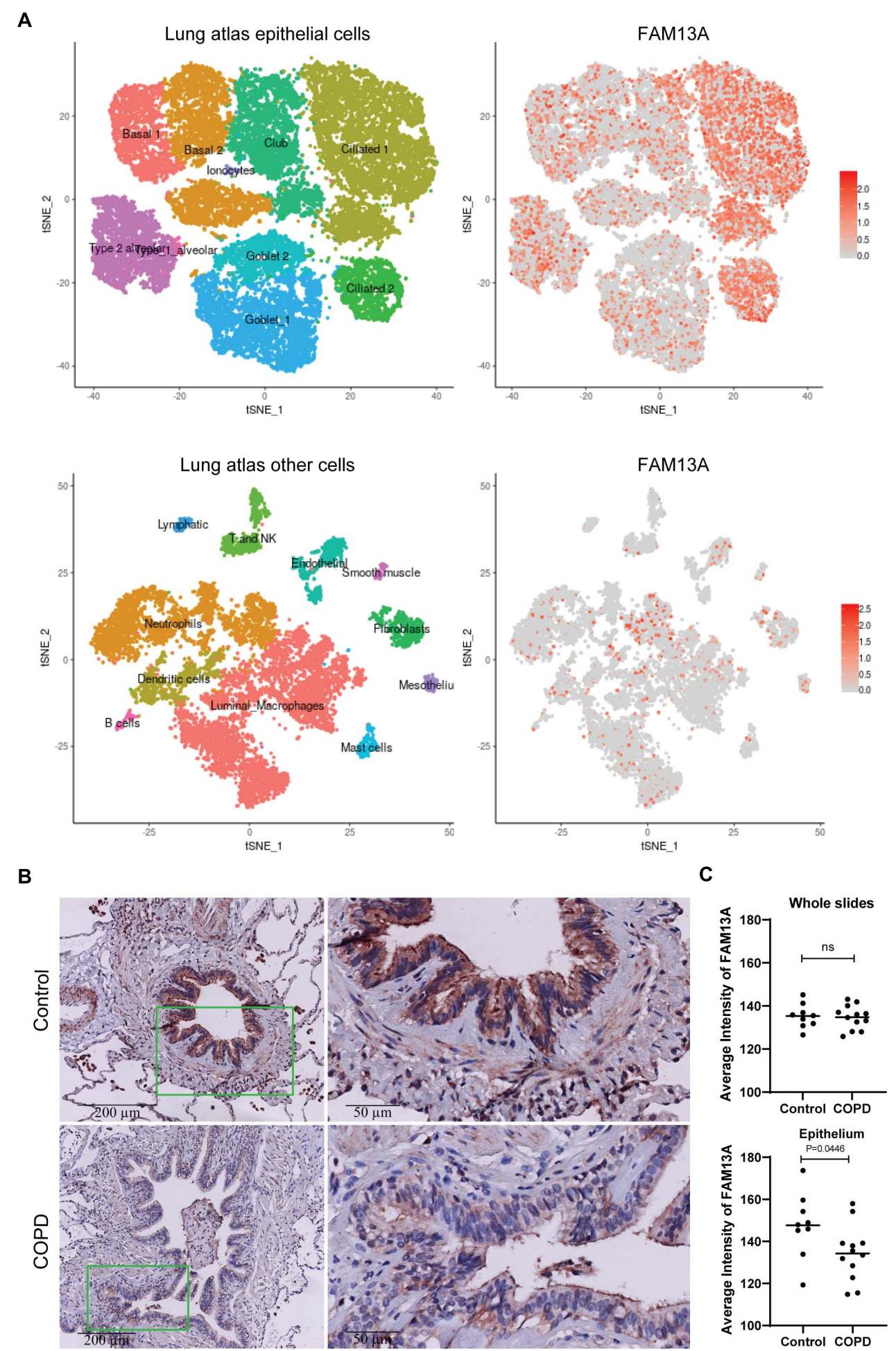

FIGURE 1 | FAM13A protein expression in human lung tissue. (A) Expression of FAM13A in single cell RNA sequencing data of airway epithelial cells. The figure in the upper left corner shows the different clusters of airway epithelial cells. The red dots on the right figures indicate the expression of FAM13A in the different clusters of the lung cells. Figures in the lower panel show the non-epithelial cell clusters and the FAM13A expression among these clusters. (B) FAM13A expression in whole lung tissue and in airway epithelial cells was assessed in 9 non-COPD controls (top) and 12 COPD patients (bottom) by immunohistochemistry (IHC). Representative images are shown. (C) Intensity of FAM13A in the whole slide and in the epithelium of every airway in the tissue. The average intensity of all airways per donor is presented. Each dot represents one subject. Data are shown as mean. Significant differences were assessed by Unpaired $t$-test and $p$-value is indicated. 
A
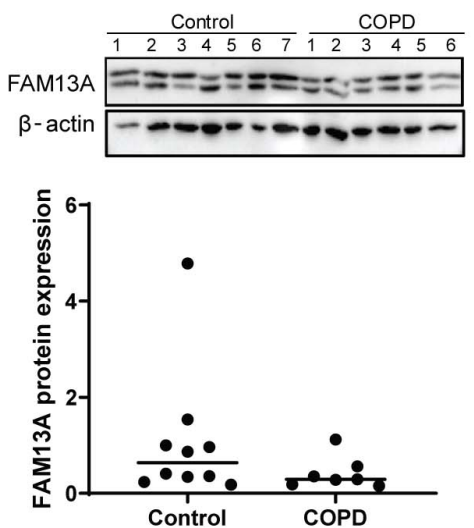

B

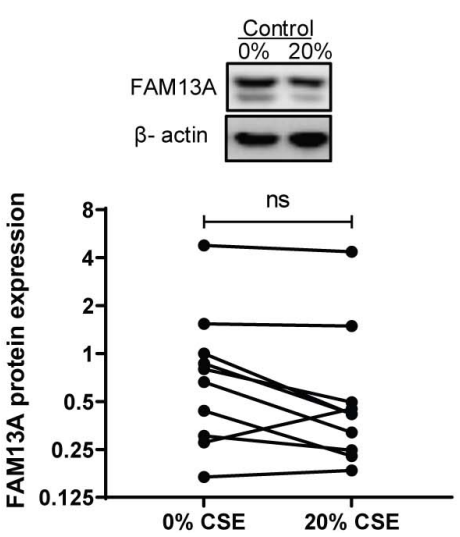

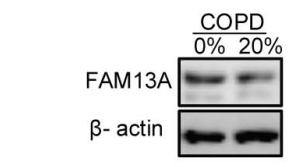

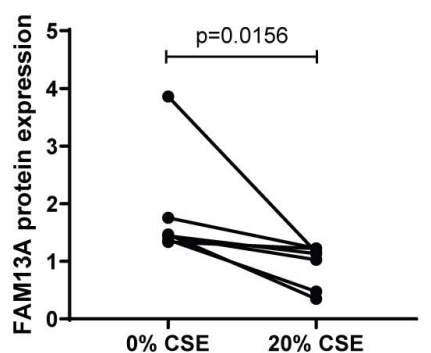

FIGURE 2 | FAM13A expression in primary human airway epithelial cells. (A) Control and COPD-derived AECs were seeded in duplicate, grown to confluence, and incubated with hormone-free media for $24 \mathrm{~h}$. FAM13A levels were detected by western blot in total cell lysates, quantified by densitometry and normalized to $\beta$-actin as the loading control. Medians are indicated, control $n=10$, COPD $n=7$. A representative blot is shown. Significant differences were assessed by the Mann-Whitney test. (B) Control and COPD-derived AECs were cultured and hormonally deprived as described above, and incubated with or without $20 \%$ cigarette smoke extract (CSE) for $24 \mathrm{~h}$. FAM13A levels were detected and quantified as for panel A. Each dot represents one subject, control $n=10$, COPD $n=7$.

Representative blots are shown. Significant differences were assessed by the Wilcoxon signed rank test and $p$-value is indicated.

\section{Increased Junctional Protein Levels Upon Overexpression of FAM13A in 16HBE140-Cells}

E-cadherin-mediated junctions are crucial for epithelial barrier formation (Nawijn et al., 2011) and are stabilized by $\beta$-catenin. We found that FAM13A overexpression in 16HBE14o- cells
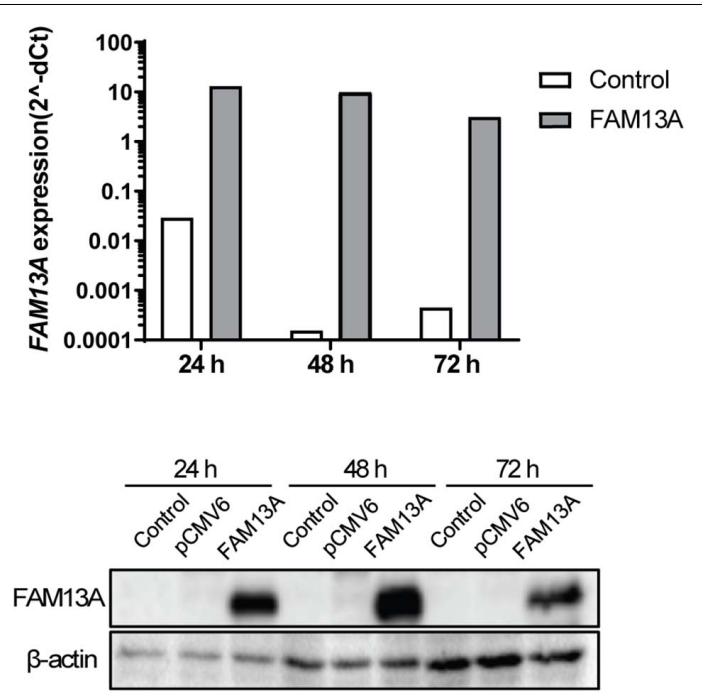

FIGURE 3 | Overexpression of FAM13A in 16HBE140- cells. 16HBE140- cells were seeded in duplicate, transfected with or without FAM13A overexpression plasmid and harvested at 24, 48, and $72 \mathrm{~h}$ after the transfection. FAM13A overexpression was confirmed at mRNA level (top) and protein level (bottom). FAM13A mRNA expression levels were measured by qPCR, corrected for the housekeeping genes B2M and PPIA and normalized to control $\left(2^{-\Delta \mathrm{Ct}}\right)$. FAM13A protein expression levels were measured using western blot. $\beta$-actin was used as the loading control. A representative blot is shown. significantly increased the expression of E-cadherin at $24 \mathrm{~h}$ (Figure 5A). We observed a similar trend for total $\beta$-catenin (Figure 5B), while the expression of active $\beta$-catenin was not affected as indicated by active/non-phospho $\beta$-catenin levels (Figure 5C). Lower expression of E-cadherin has previously been observed in COPD (Oldenburger et al., 2014). Interestingly, we found a positive correlation between expression of FAM13A and E-cadherin in lung tissue, which supports a supposed role of FAM13A in the regulation of E-cadherin expression (Figure 5D).

\section{Cigarette Smoke-Induced CXCL8 Secretion Upon FAM13A Overexpression in 16HBE140-Cells}

Finally, we investigated whether overexpression of FAM13A is able to reduce cigarette smoke-induced CXCL8 secretion. We used cigarette smoke in a concentration of $10 \%$, as the higher concentration of $20 \%$ was shown to induce cell death in the 16HBE140- cell line (Supplementary Figure 2). We observed that exposure to $10 \%$ CSE induced a significant increase in CXCL8 secretion in cells transfected with empty vector control pCMV6 (Figure 6A). Upon overexpression of FAM13A, the baseline secretion of CXCL8 was not significantly affected, but the CSE-induced CXCL8 release was significantly reduced compared to pCMV6 (Figure 6B).

\section{DISCUSSION}

In this study, we investigated the functional role of the COPD susceptibility gene FAM13A in airway epithelial barrier integrity and pro-inflammatory responses. Our data show that the intensity of FAM13A protein expression is significantly lower in the airway epithelium of COPD patients compared to non-COPD controls. In cultured airway epithelial cells, 
A

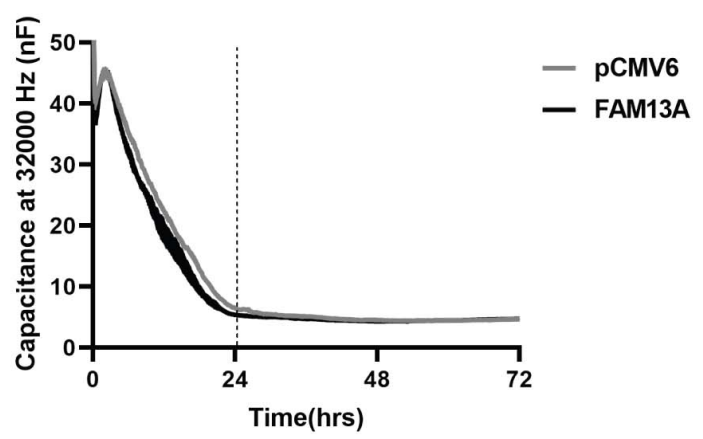

C
B

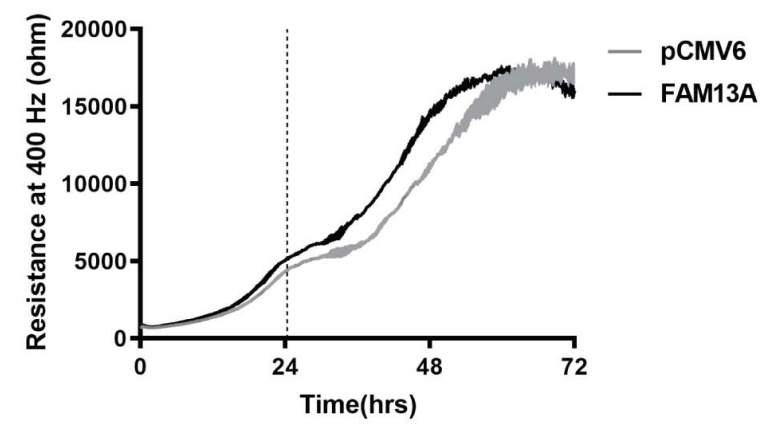

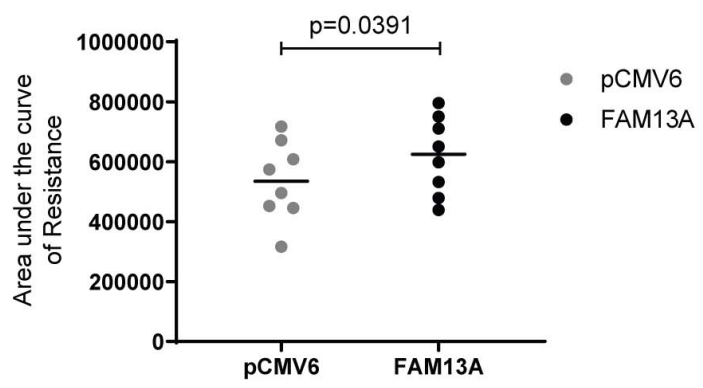

FIGURE 4 | Significantly more rapid building-up of electrical resistance upon FAM13A overexpression in 16HBE140- cells. 16HBE140- cells were transfected with FAM13A overexpression plasmid or empty vector (pCMV6). After $24 \mathrm{~h}$, the cells were trypsinized and seeded in duplicates into ECIS arrays. The high-frequency capacitance and low-frequency resistance were monitored over $72 \mathrm{~h}$ after seeding. (A) High-frequency capacitance and (B) low-frequency resistance values of a representative ECIS experiment. The vertical line indicates the time when cells reached confluence as indicated by the stabilization of high-frequency capacitance. Data are shown as mean \pm SEM of two duplicates in the ECIS array. (C) The area under the curve (AUC) of resistance values from 0 to $72 \mathrm{~h}$ was calculated and depicted as mean and individual experiments $(n=8)$. Significant differences were assessed by the Wilcoxon signed rank test and $p$-value is indicated.

FAM13A protein expression was downregulated upon CSE exposure in COPD-derived but not in control-derived cultures. Overexpression of FAM13A led to more rapid constitution of cell-cell contacts, which may be the consequence of increased junctional E-cadherin expression. In addition, overexpression of FAM13A reduced CSE-induced CXCL8 secretion, suggesting an anti-inflammatory role. Overall, our results suggest a protective role of FAM13A in the airway epithelium with respect to the features of COPD and cigarette smoke leads to lower FAM13A levels in COPD.

FAM13A was identified in multiple GWA studies on lung function and COPD (Hobbs et al., 2017). Our group previously identified a single nucleotide polymorphism (SNP) located in gene FAM13A to be negatively associated with lung function in never-smokers. Additional, eQTL analysis showed that this SNP is associated with higher gene expression of FAM13A in human lung tissue (van der Plaat et al., 2017). These previous findings suggest higher levels of FAM13A in COPD lungs, which is partly in line with the findings of Jiang et al. (2016) who found higher FAM13A protein, but not mRNA levels in lung tissue of COPD patients compared to healthy exsmokers. They suggest a detrimental instead of a protective role of FAM13A in the onset or severity of COPD, which is in contrast to our findings. However, their observations were done by western blotting in whole lung tissue, where FAM13A is also expressed in other cell types than the airway epithelium. For instance, an increase in smooth muscle mass could result in higher FAM13A expression in stromal cells, accounting for the differences observed between COPD and control. When assessing FAM13A in whole lung tissue, we did not observe a significant difference between COPD patients and controls. However, when specifically assessing the expression of FAM13A in the airway epithelium, we observed lower expression in COPD patients compared to non-COPD controls. In line, in airway epithelial cultures from COPD patients, but not in controls, we observed that smoke exposure significantly decreased FAM13A expression. Potentially, there is an interaction between the FAM13A gene and exposure to cigarette smoke, resulting in differential expression upon smoking, especially in epithelial cells which first encounter cigarette smoke. However, further studies are required to study this potential interaction in bronchial epithelium of smoking individuals. Together with the lower expression of FAM13A in COPD airway epithelium, our data suggest that cigarette smoking results in lower FAM13A expression in airway epithelial cells in COPD, subsequently leading to impaired epithelial barrier recovery and increased CXCL8 release, a key attractant of neutrophils.

Our findings that FAM13A advanced the formation of the epithelial barrier may reflect improved recovery of cell-cell contacts, supporting reinforcement of the epithelial barrier 
A
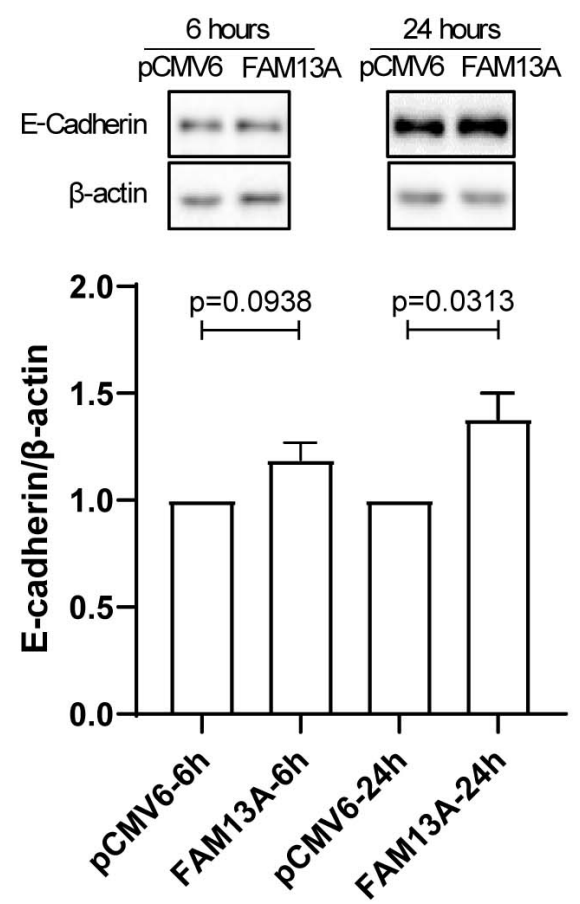

C
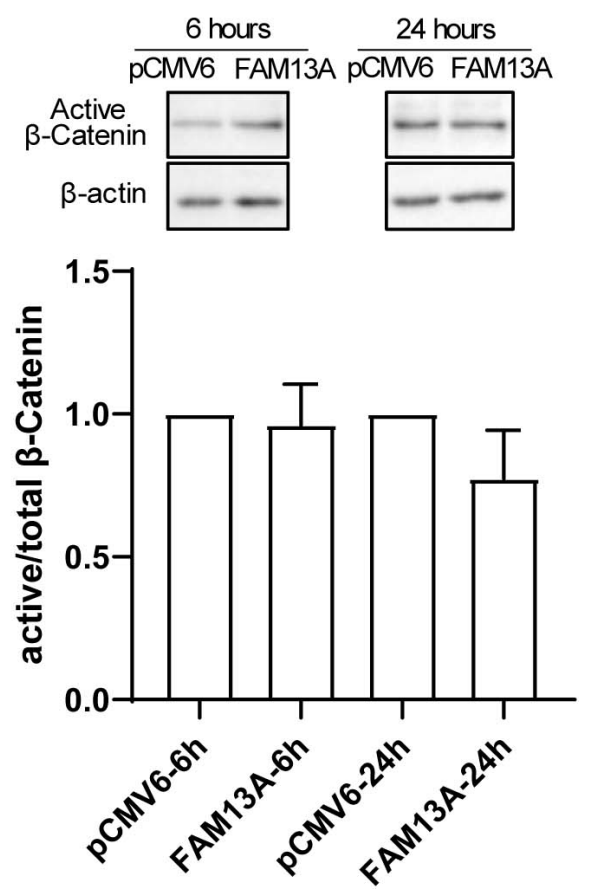

B
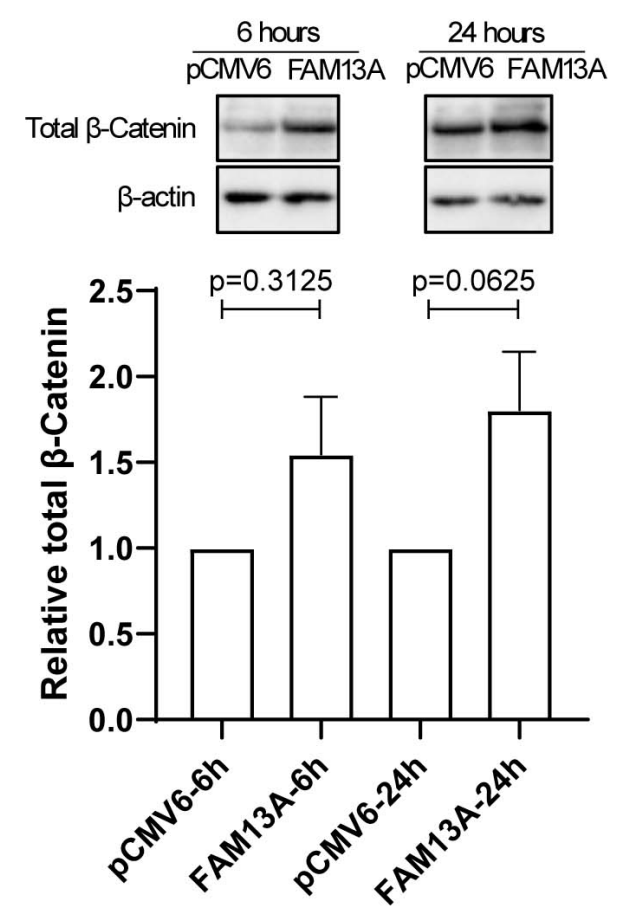

D

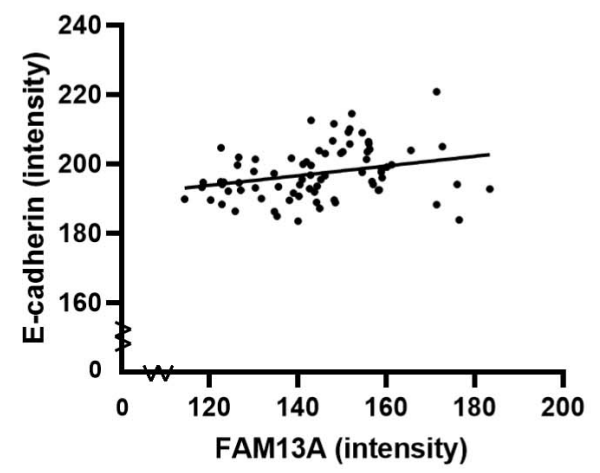

FIGURE 5 | Expression of adherens junction protein E-cadherin is increased upon overexpression of FAM13A in 16HBE140- cells and correlates with FAM13A in human lung tissue. 16HBE14o- cells were seeded in duplicate, transfected with FAM13A overexpression plasmid or empty vector (pCMV6) and harvested at 6 and $24 \mathrm{~h}$ after transfection. (A) E-cadherin protein expression levels were measured using western blot, quantified by densitometry and normalized to $\beta$-actin as loading control (mean \pm SEM, $n=6$ ). Total $\beta$-catenin (B) and active/non-phospho- $\beta$-catenin expression levels were assessed by western blot, quantified by densitometry and normalized to $\beta$-actin as loading control. (C) The ratio of active/non-phospho- $\beta$-catenin to total $\beta$-catenin is depicted. Representative blots are shown. Significant differences were assessed by the Wilcoxon signed rank test and the $p$-value between significant different conditions is as indicated. (D) The expression of E-cadherin in human lung tissue was assessed by immunohistochemistry comparable to FAM13A as described above. The correlation between the expression of FAM13A and E-cadherin in the same airway was tested using the Pearson's correlation test. Each dot represents one airway. Number of data pairs $=77, r=0.2812, p=0.0132$. 
A

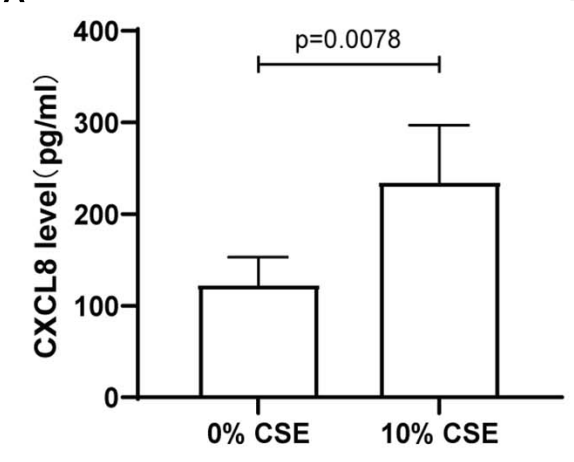

B

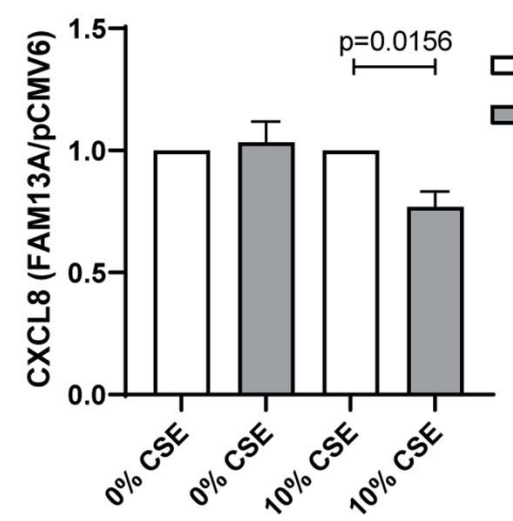

FIGURE 6 | Suppressive effect of FAM13A on CSE-induced CXCL8 secretion in 16HBE140- cells. 16HBE140- cells were seeded in duplicate, transfected with FAM13A overexpression plasmid or empty vector (pCMV6), serum deprived overnight and exposed to $10 \%$ CSE for 24 h. CXCL8 secretion levels were measured using ELISA. (A) Absolute CXCL8 levels in pCMV6 transfected cells upon CSE exposure. (B) CXCL8 secretion levels of FAM13A overexpressing cells were measured as above and normalized to the levels of pCMV6 cells of each CSE concentration, respectively, $(n=8)$. Data are shown as mean \pm SEM $(n=8)$. Significant differences were assessed by the Wilcoxon signed rank test and the $p$-value is indicated.

upon environmental insults. These effects may be explained by effects on $\beta$-catenin stability associated with higher E-cadherin expression. $\beta$-catenin is localized at the cytoplasmic side of adherens junctions and stabilizes E-cadherin (Hartsock and Nelson, 2008; Heijink et al., 2010b), while E-cadherin mediated cell-cell contacts are crucial for the formation of other epithelial junctions. Upon loss of E-cadherin, $\beta$-catenin is liberated from adherens junctions and translocated to the nucleus, activating transcription of various genes, including CXCL8 (Lévy et al., 2002; Heijink et al., 2014), but only when its phosphorylation and subsequent degradation is prevented. However, active/nonphosphorylated levels of $\beta$-catenin were not affected by FAM13A overexpression, which may indicate that $\beta$-catenin was mainly localized at epithelial junctions. It has been shown that both adherens and tight junctions are disrupted in airway epithelial cells from severe COPD patients compared to control subjects (Heijink et al., 2014; Oldenburger et al., 2014), suggesting an impaired epithelial barrier function in COPD patients. This may be due to an intrinsic deficiency to reconstitute cell-cell contacts upon epithelial damage, and lower expression of FAM13A may contribute to impaired recovery of cell-cell contacts. We speculate that FAM13A increased junctional-localized $\beta$-catenin and the expression of E-cadherin, which subsequently benefits the formation of adherens junctions and may thus advance the formation and/or recovery of the epithelial barrier. Such a role was supported by the positive correlation between FAM13A and E-cadherin expression in the airway epithelium in lung tissue.

Loss of epithelial barrier function may increase cell vulnerability to inhaled toxins such as cigarette smoke and lead to cellular damage, increased oxidative stress and subsequent release of neutrophil attractant CXCL8 (Heijink et al., 2013). Given the involvement of FAM13A in the regulation of oxidative stress and mitochondrial function (Hawkins and Mora, 2017; Jiang et al., 2017), we assessed the effects of FAM13A overexpression on CXCL8 release. We observed that
FAM13A overexpression reduced the CSE-induced increase of CXCL8 levels in 16HBE14o- cells. Thus, FAM13A may suppress pro-inflammatory responses to cigarette smoking in airway epithelium and subsequently reduce neutrophilic infiltration.

A limitation of our study is that the functional studies were performed in the cell line 16HBE140-, because of the limited availability of primary cells. However, we have previously shown that this cell line models the pro-inflammatory response of primary cells accurately (Heijink et al., 2013). Furthermore, 16HBE14o- cells form strong cell-cell contacts, which makes them highly suitable to study epithelial barrier function (Heijink et al., 2010a). In addition, 16HBE14o- express low endogenous levels of FAM13A protein, which makes them suitable for overexpression studies. Another limitation of the current study is the use of submerged epithelial cultures to assess effects of cigarette smoke extract on FAM13A expression instead of using air-liquid interface cultures exposed to gaseous-phase cigarette smoke. It is difficult to assess the most relevant method of cigarette smoke exposure. Nevertheless, previous studies from our group have shown that lipid-soluble compounds present in CSE, which we speculate to potentially represent components present in epithelial lining fluid, include aldehydes, one of the most toxic compounds of CS. These lipid-soluble components were shown to be responsible for the generation of reactive oxygen species within the epithelial cells and induction of mitochondrial damage (Hoshino et al., 2001; van der Toorn et al., 2009).

In conclusion, we found lower expression of FAM13A in airway epithelium of COPD patients. This may contribute to the pathogenesis of the disease, as FAM13A was shown to have a protective role in human airway epithelium, advancing the formation of intercellular contacts and suppressing epithelial pro-inflammatory responses upon cigarette smoke exposure. This study sheds new light on the function of FAM13A in the development of COPD and translates genetic associations to 
potential biological function, identifying FAM13A as a potential target for intervention.

\section{DATA AVAILABILITY STATEMENT}

The raw data supporting the conclusions of this article will be made available by the authors, without undue reservation.

\section{ETHICS STATEMENT}

Ethical review and approval was not required for the study on human participants in accordance with the local legislation and institutional requirements. Written informed consent for participation was not required for this study in accordance with the national legislation and the institutional requirements.

\section{AUTHOR CONTRIBUTIONS}

QC, MV, HB, and IH contributed to the conception and design of the study. QC, MV, KON, and JN performed the experiments. $\mathrm{C}$-AB supplied the lung tissue, and involved in formatting the protocol of staining and image analysis. QC, MV, and $\mathrm{IH}$ formatted analysis and interpretation and wrote the first draft of the manuscript. All authors contributed to manuscript revision, read, and approved the submitted version.

\section{REFERENCES}

Abrams, D. B., Alberg, A. J., Anderson, G. P., Ardaugh, B. M., Baumgartner, K. B., Baumgartner, R. N., et al. (2014). The Health Consequences of Smoking-50 Years of Progress A Report of the Surgeon General. Atlanta, GA: Centers for Disease Control and Prevention (US).

Adeloye, D., Chua, S., Lee, C., Basquill, C., Papana, A., Theodoratou, E., et al. (2015). Global and regional estimates of COPD prevalence: systematic review and meta-analysis. J. Glob. Health 5:020415. doi: 10.7189/jogh.05.020415

Aghapour, M., Raee, P., Moghaddam, S. J., Hiemstra, P. S., and Heijink, I. H. (2018). Airway epithelial barrier dysfunction in chronic obstructive pulmonary disease: role of cigarette smoke exposure. Am. J. Respir. Cell Mol. Biol. 58, 157-169. doi: 10.1165/rcmb.2017-0200TR

Aghapour, M., Remels, A. H. V., Pouwels, S. D., Bruder, D., Hiemstra, P. S., Cloonan, S. M., et al. (2020). Mitochondria: at the crossroads of regulating lung epithelial cell function in chronic obstructive pulmonary disease. Am. J. Physiol. Lung Cell. Mol. Physiol. 318, L149-L164. doi: 10.1152/ajplung.00329.2019

Barnes, P. J. (2008). Immunology of asthma and chronic obstructive pulmonary disease. Nat. Rev. Immunol. 8, 183-192. doi: 10.1038/nri2254

Barnes, P. J., Shapiro, S. D., and Pauwels, R. A. (2003). Chronic obstructive pulmonary disease: molecular and cellular mechanisms. Eur. Respir. J. 22, 672-688. doi: 10.1183/09031936.03.00040703

Brusselle, G. G., Joos, G. F., and Bracke, K. R. (2011). New insights into the immunology of chronic obstructive pulmonary disease. Lancet 378, 1015-1026. doi: 10.1016/S0140-6736(11)60988-4

Di Vincenzo, S., Heijink, I. H., Noordhoek, J. A., Cipollina, C., Siena, L., Bruno, A., et al. (2018). SIRT1/FoxO3 axis alteration leads to aberrant immune responses in bronchial epithelial cells. J. Cell. Mol. Med. 22, 2272-2282. doi: 10.1111/ jcmm.13509

Gangl, K., Reininger, R., Bernhard, D., Campana, R., Pree, I., Reisinger, J., et al. (2009). Cigarette smoke facilitates allergen penetration across respiratory epithelium. Allergy Eur. J. Allergy Clin. Immunol. 64, 398-405. doi: 10.1111/j. 1398-9995.2008.01861.x

\section{FUNDING}

This work was supported by the China Scholarship Council (No. 201706300019) and the University Medical Center Groningen.

\section{ACKNOWLEDGMENTS}

We would like to thank M.Sc. Marijn Berg for providing high resolution figures of the single cell sequence data. The stainings of FAM13A and E-cadherin on lung tissue performed in this manuscript were conducted as part of the HOLLAND (HistopathOLogy of Lung Aging aNd COPD) project. The HOLLAND project was initiated and supervised by C-AB, Wim Timens, and Janette Burgess, technical support was provided by Marjan Reinders-Luinge, Anja Bakker, and Theo Borghuis, and image analyses pipelines were developed by Theo Borghuis, Maunick Lefin Koloko Ngassie, and Niek Bekker. We would like to thank them for their support on the immunohistochemistry data.

\section{SUPPLEMENTARY MATERIAL}

The Supplementary Material for this article can be found online at: https://www.frontiersin.org/articles/10.3389/fphys. 2021.690936/full\#supplementary-material

Hartsock, A., and Nelson, W. J. (2008). Adherens and tight junctions: structure, function and connections to the actin cytoskeleton. Biochim. Biophys. Acta Biomembr. 1778, 660-669. doi: 10.1016/j.bbamem.2007.07.012

Hawkins, G. A., and Mora, A. L. (2017). FAM13A, a fatty acid oxidation switch in mitochondria. friend or foe in chronic obstructive pulmonary disease pathogenesis? Am. J. Respir. Cell Mol. Biol. 56, 689-691. doi: 10.1165/rcmb. 2017-0080ED

Heijink, I. H., Brandenburg, S. M., Noordhoek, J. A., Postma, D. S., Slebos, D. J., and van Oosterhout, A. J. M. (2010a). Characterisation of cell adhesion in airway epithelial cell types using electric cell-substrate impedance sensing. Eur. Respir. J. 35, 894-903. doi: 10.1183/09031936.00065809

Heijink, I. H., Brandenburg, S. M., Postma, D. S., and van Oosterhout, A. J. M. (2012). Cigarette smoke impairs airway epithelial barrier function and cell-cell contact recovery. Eur. Respir. J. 39, 419-428. doi: 10.1183/09031936.00193810

Heijink, I. H., De Bruin, H. G., Dennebos, R., Jonker, M. R., Noordhoek, J. A., Brandsma, C. A., et al. (2016). Cigarette smoke-induced epithelial expression of WNT-5B: implications for COPD. Eur. Respir. J. 48, 504-515. doi: 10.1183/ 13993003.01541-2015

Heijink, I. H., De Bruin, H. G., van den Berge, M., Bennink, L. J. C., Brandenburg, S. M., Gosens, R., et al. (2013). Role of aberrant WNT signalling in the airway epithelial response to cigarette smoke in chronic obstructive pulmonary disease. Thorax 68, 709-716. doi: 10.1136/thoraxjnl-2012-201667

Heijink, I. H., Kies, P. M., Kauffman, H. F., Postma, D. S., van Oosterhout, A. J. M., and Vellenga, E. (2007). Down-regulation of E-cadherin in human bronchial epithelial cells leads to epidermal growth factor receptor-dependent Th2 cellpromoting activity. J. Immunol. 178, 7678-7685. doi: 10.4049/jimmunol.178. 12.7678

Heijink, I. H., Noordhoek, J. A., Timens, W., van Oosterhout, A. J. M. M., and Postma, D. S. (2014). Abnormalities in airway epithelial junction formation in chronic obstructive pulmonary disease. Am. J. Respir. Crit. Care Med. 189, 1439-1442. doi: 10.1164/rccm.201311-1982LE

Heijink, I. H., Postma, D. S., Noordhoek, J. A., Broekema, M., and Kapus, A. (2010b). House dust mite-promoted epithelial-to-mesenchymal transition in 
human bronchial epithelium. Am. J. Respir. Cell Mol. Biol. 42, 69-79. doi: 10.1165/rcmb.2008-0449OC

Hellings, P. W., and Steelant, B. (2020). Epithelial barriers in allergy and asthma. J. Allergy Clin. Immunol. 145, 1499-1509. doi: 10.1016/j.jaci.2020.04.010

Higham, A., Bostock, D., Booth, G., Dungwa, J. V., and Singh, D. (2018). The effect of electronic cigarette and tobacco smoke exposure on COPD bronchial epithelial cell inflammatory responses. Int. J. Chron. Obstruct. Pulmon. Dis. 13, 989-1000. doi: 10.2147/COPD.S157728

Hobbs, B. D., De Jong, K., Lamontagne, M., Bossé, Y., Shrine, N., Artigas, M. S., et al. (2017). Genetic loci associated with chronic obstructive pulmonary disease overlap with loci for lung function and pulmonary fibrosis. Nat. Genet. 49, 426-443. doi: 10.1038/ng.3752

Hoshino, Y., Mio, T., Nagai, S., Miki, H., Ito, I., and Izumi, T. (2001). Cytotoxic effects of cigarette smoke extract on an alveolar type II cell-derived cell line. Am. J. Physiol. Lung Cell. Mol. Physiol. 281, L509-L516. doi: 10.1152/ajplung. 2001.281.2.1509

Jiang, Z., Knudsen, N. H., Wang, G., Qiu, W., Naing, Z. Z. C., Bai, Y., et al. (2017). Genetic control of fatty acid b-oxidation in chronic obstructive pulmonary disease. Am. J. Respir. Cell Mol. Biol. 56, 738-748. doi: 10.1165/rcmb.2016$0282 \mathrm{OC}$

Jiang, Z., Lao, T., Qiu, W., Polverino, F., Gupta, K., Guo, F., et al. (2016). A chronic obstructive pulmonary disease susceptibility gene, FAM13A, regulates protein stability of $\beta$-catenin. Am. J. Respir. Crit. Care Med. 194, 185-197. doi: 10.1164/rccm.201505-0999OC

Lévy, L., Neuveut, C., Renard, C. A., Charneau, P., Branchereau, S., Gauthier, F., et al. (2002). Transcriptional activation of interleukin- 8 by $\beta$-catenin-Tcf 4 . J. Biol. Chem. 277, 42386-42393. doi: 10.1074/jbc.M207418200

Meng, W., and Takeichi, M. (2009). Adherens junction: molecular architecture and regulation. Cold Spring Harb. Perspect. Biol. 1:a002899. doi: 10.1101/ cshperspect.a002899

Nawijn, M. C., Hackett, T. L., Postma, D. S., van Oosterhout, A. J. M., and Heijink, I. H. (2011). E-cadherin: gatekeeper of airway mucosa and allergic sensitization. Trends Immunol. 32, 248-255. doi: 10.1016/j.it.2011.03.004

Oldenburger, A., Poppinga, W. J., Kos, F., de Bruin, H. G., Rijks, W. F., Heijink, I. H., et al. (2014). A-kinase anchoring proteins contribute to loss of E-cadherin and bronchial epithelial barrier by cigarette smoke. Am. J. Physiol. Cell Physiol. 306, C585-C597. doi: 10.1152/ajpcell.00183.2013

Pouwels, S. D., Zijlstra, G. J., van der Toorn, M., Hesse, L., Gras, R., Hacken, N. H. T. T., et al. (2016). Cigarette smoke-induced necroptosis and DAMP release trigger neutrophilic airway inflammation in mice. Am. J. Physiol. Lung Cell. Mol. Physiol. 310, L377-L386. doi: 10.1152/ajplung.00174.2015

Ruifrok, A. C., and Johnston, D. A. (2001). Quantification of histochemical staining by color deconvolution. Anal. Quant. Cytol. Histol. 23, 291-299.

Schindelin, J., Arganda-Carreras, I., Frise, E., Kaynig, V., Longair, M., Pietzsch, T., et al. (2012). Fiji: an open-source platform for biological-image analysis. Nat. Methods 9, 676-682. doi: 10.1038/nmeth.2019

van der Plaat, D. A., de Jong, K., Lahousse, L., Faiz, A., Vonk, J. M., van Diemen, C. C., et al. (2017). Genome-wide association study on the FEV1/FVC ratio in never-smokers identifies HHIP and FAM13A. J. Allergy Clin. Immunol. 139, 533-540. doi: 10.1016/j.jaci.2016.06.062

van der Toorn, M., Rezayat, D., Kauffman, H. F., Bakker, S. J. L., Gans, R. O. B., Koëter, G. H., et al. (2009). Lipid-soluble components in cigarette smoke induce mitochondrial production of reactive oxygen species in lung epithelial cells. Am. J. Physiol. Lung Cell. Mol. Physiol. 297, L109-L114. doi: 10.1152/ajplung.90461. 2008

van der Vaart, H., Postma, D. S., Timens, W., Hylkema, M. N., Willemse, B. W. M., Boezen, H. M., et al. (2005). Acute effects of cigarette smoking on inflammation in healthy intermittent smokers. Respir. Res. 6:22. doi: 10.1186/1465-9921-6-22

Vieira Braga, F. A., Kar, G., Berg, M., Carpaij, O. A., Polanski, K., Simon, L. M., et al. (2019). A cellular census of human lungs identifies novel cell states in health and in asthma. Nat. Med. 25, 1153-1163. doi: 10.1038/s41591-0190468-5

Wegener, J., Keese, C. R., and Giaever, I. (2000). Electric cell-substrate impedance sensing (ECIS) as a noninvasive means to monitor the kinetics of cell spreading to artificial surfaces. Exp. Cell Res. 259, 158-166. doi: 10.1006/excr.2000. 4919

Conflict of Interest: The authors declare that the research was conducted in the absence of any commercial or financial relationships that could be construed as a potential conflict of interest.

Copyright (c) 2021 Chen, de Vries, Nwozor, Noordhoek, Brandsma, Boezen and Heijink. This is an open-access article distributed under the terms of the Creative Commons Attribution License (CC BY). The use, distribution or reproduction in other forums is permitted, provided the original author(s) and the copyright owner(s) are credited and that the original publication in this journal is cited, in accordance with accepted academic practice. No use, distribution or reproduction is permitted which does not comply with these terms. 